\title{
Some notes on metric and fuzzy metric spaces
}

Saleh Omran ${ }^{1,2, *}$, H. S. Al-Saadi ${ }^{3}$

${ }_{1}^{1}$ Mathematics Department, Faculty of Science, South Valley University, Qena, Egypt

${ }^{2}$ Mathematics Department, Faculty of Science, Taif University, Taif, Saudi Arabia

${ }^{3}$ Mathematics Department, Faculty of Applied Sciences, Umm Al-Qura University, Makkah 21955, P. O. Box 11155, Saudi Arabia

\section{A R T I C L E I N F O}

Article history:

Received 11 December 2016

Received in revised form

6 March 2017

Accepted 4 April 2017

\section{Keywords:}

Metric spaces

Fuzzy metric spaces

Fixed point

\begin{abstract}
A B S T R A C T
In this paper we define the fuzzy metric space by using the usual definition of the metric space and vise versa, so we can obtain each one from the other. We prove some fixed point theorems on the fuzzy metric spaces.
\end{abstract}

(C) 2017 The Authors. Published by IASE. This is an open access article under the CC BY-NC-ND license (http://creativecommons.org/licenses/by-nc-nd/4.0/).

\section{Introduction}

In 1906, the French mathematician Maurice Frechet introduced the concept of metric spaces; metric space is a very important subject in analysis and topology (Fréchet, 1906). In 1965, the concept of fuzzy set was introduced by Zadeh (1965). Many authors have introduced different ways of the concept of fuzzy metric space (Erceg, 1979; Diamond and Kloden, 1999; George and Veeramani, 1994) and (Gregori and Romaguera, 2000). In this work we give a definition of the fuzzy metric space by using the ordinary metric and vise versa.

\section{Preliminaries}

In this section, we recall some basic concepts and results in both metric and fuzzy metric spaces.

Definition 2.1: A metric space is given by a set $X$ and a distance function $d: X \times X \rightarrow \mathbb{R}$ defined on $X$ such that $x, y, z \in X$ (Fréchet, 1906):

(i) $d(x, y) \geq 0, d(x y)=0 \leftrightarrow x=y$

(ii) $d(x, y)=d(y, x)$

(iii) $d(x, z) \leq d(x, y)+d(y, z)$

Definition 2.2: A fuzzy set $A$ in $X$ is a function with domain $X$ and values in $[0,1]$ (Zadeh, 1965).

Definition 2.3: A binary operation $*:[0,1]^{2} \rightarrow$ $[0,1]$ is called a continuous triangular norm (shortly

\footnotetext{
* Corresponding Author

Email Address: salehomran@yahoo.com (S. Omran)

https://doi.org/10.21833/ijaas.2017.05.007

2313-626X/C 2017 The Authors. Published by IASE.

This is an open access article under the CC BY-NC-ND license

(http://creativecommons.org/licenses/by-nc-nd/4.0/)
}

t-norm) if it satisfies the following conditions (Schweizer and Skala, 1960):

(i) * is associative and commutative,

(ii) * is continuous,

(iii) $a * 1=a$ for all $a, b, c \in[o, 1]$

(iv) $a * b \leq c * d$ whenever $\quad a \leq c$ and $\quad b \leq a$ for all $a, b, c, d \in[0,1]$

Examples of t-norm are $a * b=a b, a * b=$ $\min \{a, b\}$ and $a * b=\max \{a, b\}$.

Definition 2.4: The 3-tuple $\left(X, d_{F}, *\right)$ is called a fuzzy metric space if $X$ is an arbitrary (non-empty) set, $*$ is a continuous $t$-norm and $d_{F}$ is a fuzzy set on $X^{2} \times[0, \infty)$ satisfying the following conditions, for all $x, y, z \in X$, each $t$ and $s>0$ (George and Veeramani, 1994):

(i) $d_{F}(x, y, t)>0$

(ii) $d_{F}(x, y, t)=0$ if and only if $x=y$,

(iii) $d_{F}(x, y, t)=d_{F}(y, x, t)$,

(iv) $d_{F}(x, y, t) * d_{F}(y, z, t) \leq d_{F}(x, z, t+s)$

(v) $d_{F}(x, y, \cdot):(0, \infty) \rightarrow[0,1]$ is continuous.

Then is $d_{F}$ called a fuzzy metric on $X$. Then $d_{F}(x, y, t)$ denotes the degree of nearness between $x$ and $y$ with respect to $t$.

\section{Main results}

The main result is to obtain the fuzzy metric spaces from any ordinary metric spaces and vise versa and verify the Banach fixed point.

Proposition 3.1: Let $d$ and $d_{F}$ are metric and fuzzy metric respectively, so the following diagram

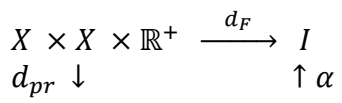


$X \times X \stackrel{d}{\longrightarrow} \mathbb{R}^{+}$

is commutative. Where, $d_{p r}:(x, y, t) \rightarrow(t x, t y)$, $d(t x, t y) \rightarrow t r$ for some metric $d(x, y)=r>0$ and $\alpha:(t r) \rightarrow 1-\frac{2}{\pi} \tan ^{-1}(t r)=: \tilde{t} \in I$. Moreover $d_{F}=\alpha \circ d \circ d_{p r}$.

Proof: It is easy to check that $\alpha$ is continuous, since $\tan ^{-1}$ in $\mathbb{R}^{+}$is continuous this implies that $\alpha$ is continuous.

Now we prove that $d \circ d_{p r}=\alpha^{-1} \circ d_{F}$. For $(x, y, z) \in X \times X \times \mathbb{R}^{+}$, we have

$d \circ d_{p r}(x, y, t)=d(t x, t y)=t r:=s>0$.

On the other side,

$\alpha^{-1} \circ d_{F}(x, y, t)=\alpha^{-1}(\tilde{t})=\alpha^{-1}\left(1-\frac{2}{\pi} \tan ^{-1}(t r)\right)=$ $\frac{\pi}{2} \tan \left[1-\left(1-\frac{2}{\pi} \tan ^{-1}(t r)\right)\right]=\operatorname{tr}$

So, the above diagram is commutative.

Lemma 3.2: Let $t_{1}, t_{2} \in \mathbb{R}^{+}$, if $t_{1} \leq t_{2}$, then $\alpha\left(t_{1}\right) \geq$ $\alpha\left(t_{2}\right)$ and $\alpha\left(t_{1}+t_{2}\right) \leq \min \left(\alpha\left(t_{1}\right), \alpha\left(t_{2}\right)\right)$.

Proof: If $t_{1} \leq t_{2}$, this implies that $\tan ^{-1} t_{1} \leq$ $\tan ^{-1} t_{2}$, this implies that $-\tan ^{-1} t_{1} \geq$ $-\tan ^{-1} t_{2}$, therefore $\quad 1-\frac{2}{\pi} \tan ^{-1} t_{1} \geq 1-$ $\frac{2}{\pi} \tan ^{-1} t_{2}$ hence $\alpha\left(t_{1}\right) \geq \alpha\left(t_{1}\right)$

The second assertion is become obvious.

Theorem 3.3: Let $(X, d)$ be the metric space and $\left(X, d_{F}, *\right)$ is a fuzzy metric space with $a * b=$ $\min \{a, b\}$ for all $a, b \in I$. Then for all , $y, z \in X$, $t, s, r \in \mathbb{R}^{+}$, we have $\left(X, \alpha \circ d \circ d_{p r}, *\right)$ is a fuzzy metric space.

Proof: We check that the conditions (i), (ii), (iii) in definition 2.4 .

For $\quad(1), \quad \alpha \circ d \circ d_{p r}(x, y, t)=\alpha \circ d(t x, t y)=$ $\alpha(d(t x, t y))=\alpha(t r)=\tilde{t}>0$.

For $\quad(2), \quad \alpha \circ d \circ d_{p r}(x, x, t)=\alpha \circ d(t x, t x)=$ $\alpha(d(t x, t x))=\alpha(0)=1$.

For $\quad(3), \quad \alpha \circ d \circ d_{p r}(x, y, t)=\alpha \circ d(t x, t y)=$ $\alpha(d(t x, t y))=\alpha(t r)=\tilde{t}$.

On the other side

$\alpha \circ d \circ d_{p r}(y, x, t)=\alpha \circ d(t y, t x)=\alpha(d(t y, t x))=$ $\alpha(t r)=\tilde{t}$

Now we check (4)

$\alpha \circ d \circ d_{p r}(x, z, t+s)=\alpha \circ d((t+s) x,(t+s) z)=$ $\alpha(d(t+s) x,(t+s) z)=\alpha((t+s) r)=\alpha(t r+s r)$

Using lemma 3.2, we have $\alpha(t r+s r) \geq \min (\alpha(t r), \alpha(s r))=\alpha(t r) * \alpha(s r)=$ $\alpha(d(t x, t y)) * \alpha(d(s y, s z))=\alpha \circ d \circ d_{p r}(x, y, t) * \alpha \circ$ $d \circ d_{p r}(y, z, s)$.

For (5) is trivial. Therefore, $\left(X, \alpha \circ d \circ d_{p r}, *\right)$ is a fuzzy metric space.

Remark 3.4: On the other side we can get the ordinary metric space from the fuzzy metric space from the above commutative diagram. So, if $\left(X, \alpha \circ d \circ d_{p r}, *\right)$ is a fuzzy metric space, then the associative metric is $\left(X, \alpha^{-1} \circ d_{F} \circ d_{p r}{ }^{-1}\right)$.

Definition 3.5: Let $(X, d)$ be a metric space on $X$, and $\left\{x_{n}\right\}$ be a sequence in $X$ then is $\left\{x_{n}\right\}$ called converge sequence to some fixed

$x \in X$ if $\exists \epsilon>o, N \in \mathbb{N}$,

$d\left(x_{n}, x\right)<\epsilon \forall n>N$

We write also $x_{n} \rightarrow x$ if $\left\{x_{n}\right\}$ converge to $x$; and $\left\{x_{n}\right\}$ is called a cauchy sequence

$d\left(x_{n}, x_{m}\right)<\epsilon \forall n, m>N$.

Definition 3.6: Let $(X, d)$ and $\left(X, d_{F}, *\right)$ are metric and fuzzy metric space on $X$, respectively. And $\left\{x_{n}\right\}$ is a sequence in $X$ then the following is equivalent.

(i) $\left\{x_{n}\right\}$ is convergent in the metric space $(X, d)$

(ii) $\mathrm{d}\left(\mathrm{x}_{\mathrm{n}}, \mathrm{x}\right)<\varepsilon \forall \mathrm{n}>\mathrm{N}$

(iii) $\left\{\mathrm{x}_{\mathrm{n}}\right\}$ is convergent in the fuzzy metric space $\left(\mathrm{X}, \alpha \circ \mathrm{d} \circ \mathrm{d}_{\mathrm{pr}}, *\right)$

(iv) For any $0<\varepsilon<1$ and $t>0$ there exists $n>N$ such that

$\alpha \circ d \circ d_{p r}\left(x_{n}, x, t\right)>1-\varepsilon$

Definition 3.7: A metric space $(X, d)$ is complete if every cauchy sequence in $X$ is convergent.

Definition 3.8: A fuzzy metric space $(X, \alpha \circ d \circ$ $\left.d_{p r}, *\right)$ is complete iff $(X, d)$ is complete.

In the following theorem we prove that if any self-map has fixed point theorems in the metric space, then it has the same fixed point theorem in the induced fuzzy metric space and vise versa. We refer to Mihet (2004) and Shen et al. (2012) for fixed point theorems in the fuzzy metric spaces.

Theorem 3.9: Let $(X, d)$ be a complete metric space on $X$, suppose the mapping $T: X \rightarrow X$ satisfy the contractive condition, thus $d(T x, T y)<k d(x, y)$ for all $x, y \in X, k \in[o, 1)$ is a constant. If $T$ has a unique fixed point in $X$ with respect to the metric $(X, d)$, then $T$ has a unique fixed point with respect to the induced fuzzy metric.

$\left(X, \alpha \circ d \circ d_{p r}, *\right)$

Proof: Suppose that $T$ has a unique fixed point in $X$ with respect to the metric space $(X, d)$. So, we have $d(T x, x)=0$ for some $x$. Therefore 
$d_{F}(T x, x, t)=\alpha \circ d \circ d_{p r}(T x, x, t)=\alpha \circ$

$d(t(T x), t(T(y))=\alpha(r t)=\alpha(0)=1$

this implies that $T x=x$ with respect to the Fuzzy metric space

$\left(X, \alpha \circ d \circ d_{p r}, *\right)$

if there another fixed point $y \in X$, then

$d_{F}(x, y, t)=\alpha \circ d \circ d_{p r}(T x, T y, t)=\alpha \circ$

$d(t(T x), t(T y))=\alpha(r t)=\alpha(0)=1$;

and therefore $x=y$.

\section{Conclusion}

In this article, we induced fuzzy metric spaces from any ordinary metric spaces and vise versa, we check that the self-map has a fixed point theorems with respect to the induced fuzzy metric spaces if it has a fixed point theorems with the ordinary metric spaces.

\section{References}

Diamond P and Kloden P (1999). Metric spaces of fuzzy sets. Fuzzy Sets and Systems, 35(2): 241-249.

Erceg MA (1979). Metric spaces in fuzzy set theory. Journal of Mathematical Analysis and Applications, 69(1): 205-230.

Fréchet MM (1906). Sur quelques points du calcul fonctionnel. Rendiconti del Circolo Matematico di Palermo (1884-1940), 22(1): 1-72.

George A and Veeramani PV (1994). On some results of fuzzy metric spaces. Fuzzy Sets and Systems, 64(3): 395-399.

Gregori V and Romaguera S (2000). Some properties of fuzzy metric spaces. Fuzzy Sets and Systems, 115(3): 485-489.

Mihet D (2004). A Banach contraction theorem in fuzzy metric spaces. Fuzzy Sets and Systems, 144(3): 431-439.

Schweizer B and Skala A (1960). Statistical metric spaces. The Pacific Journal of Mathematics, 10(1):314-334.

Shen Y, Qiu D, and Chen W (2012). Fixed point theorems in fuzzy metric spaces. Applied Mathematics Letters, 25(2): 138-141.

Zadeh LA (1965). Fuzzy sets. Information and Control, 8(3): 338353. 